\title{
Spontaneous achilles tendon rupture in a patient with undergoing long-term haemodialysis: a case report and literature review
}

\begin{abstract}
Chronic kidney disease and hemodialysis are well known risk factors for tendon rupture, although this is a rare situation. The present case was a 70-year old male who presented with a sudden onset of painful disability in the left posterior ankle. This occurred while he was climbing the stairs. A physical examination and imaging resulted in a diagnosis of Achilles tendon rupture. The patient had received regular haemodialysis for 12years. $\mathrm{He}$ developed secondary hyperparathyroidism as excepted sequelae of end stage renal disease. Following surgical repair, physiotherapy and management of associated risk factors, the patient regained full active mobility.
\end{abstract}

Volume 2 Issue 4 - 2015

\author{
Mohammed Abdel Gawad \\ Nephrology Specialist, Kidney \& Urology Center, Egypt \\ Correspondence: Mohammed Abdel Gawad, Nephrology \\ Specialist, Kidney \& Urology Center, Alexandria, Egypt, \\ Email drgawad@gmail.com
}

Received: June 10,2015 | Published: July 24, 2015

Keywords: hyperparathyroidism, haemodialysis, achilles, tendon, rupture

\section{Introduction}

Cases of simultaneous Achilles tendon rupture in patients on regular hemodialysis have been described in the literature. ${ }^{1-3}$ Many risk factors in hemodialysis patients may contribute to this presentation, but secondary hyperparathyroidism plays a major role in the pathogenesis of tendon rupture. ${ }^{4}$ Early treatment of ruptured tendons is mandatory as it results in better outcomes and prevents disabilities. This current report presents a case of spontaneous Achilles tendon rupture in a patient undergoing long term haemodialysis, with multiple risk factors predisposing to this condition.

\section{Case report}

A 70years old male patient, who was on maintenance hemodialysis ( 3 times per week) since 12years, presented to us with a sudden onset of painful disability in the left posterior ankle developed while he was climbing the stairs. On examination, by inspection there was swelling around left posterior ankle joint. On palpation there was tenderness above the insertion of the Achilles tendon, also there was a gap in the Achilles tendon site. Thompson calf squeeze test was positive. Ultrasonography revealed a complete disruption of the fibrillar structure of Achilles tendon. The serum parathyroid hormone level of the patient was $410 \mathrm{pg} / \mathrm{ml}$ (normal range $15-65 \mathrm{pg} / \mathrm{ml}$ ). Based on the previous findings and data, a diagnosis of Achilles tendon rupture was confirmed with associated secondary hyperparathyroidism.

A decision of surgical repair was taken. During exploration, a tear at the lower third of Achilles tendon was detected. The color of the tissue stump was dark brown; also the tendon was degenerative and weak. The tendon was repaired by non-absorbable mono-filamentous sutures. A short leg cast was postoperatively applied with foot in gravity equinus i.e. non-bearing cast. Every 3 weeks we performed a sequential change of the cast shape and foot position with re-casting in a more dorsiflexion position each time till a 90degree position cast reached after the sixth week. The patient had been in the 90degree position cast for another two weeks, and then cast was removed. Physiotherapy started after cast removal, and the patient almost completely regained his normal ankle function 2 months later.
Parallel to surgical repair and physiotherapy we started to manage secondary hyperparathyroidism and high parathyroid hormone level using calcium supplements, phosphate binders and vitamin D analogues according to his laboratory result trends. His serum parathyroid hormone level was $300 \mathrm{pg} / \mathrm{ml}$ after this tight control, which is accepted level regarding the recommended targets by KDIGO (Kidney Disease-Improving Global Outcomes).

\section{Discussion}

Tendinopathy is prominent in hemodialysis patients, ${ }^{5}$ but the presentation with tendon rupture is rare. Tendon rupture has been described as a complication of multiple disorders rather than chronic kidney disease and hemodialysis, ${ }^{6}$ as systemic lupus erythematosus, ${ }^{7}$ gout, ${ }^{8}$ rheumatoid arthritis, ${ }^{9}$ diabetes mellitus, ${ }^{10}$ obesity ${ }^{11}$ and trauma.

The most frequently affected tendons are quadriceps tendon, patellar tendon, and Achilles tendon. ${ }^{1,3}$ Also a more rare reported case of rupture pectoralis major muscle was reported in a hemodialysis patient. ${ }^{12}$ The first reported ruptured tendon in hemodialysis patient was in 1949, and it was a case of simultaneous bilateral rupture of the quadriceps tendon. ${ }^{13}$

Chronic kidney disease and hemodialysis sequelae are suggested to be the predisposing factors for rupture beside other concomitant factors. These predisposing factors include being on long-term hemodialysis, development of secondary hyperparathyroidism, b-2microglobulin associated amyloidosis, fluoroquinolone use, corticosteroid use, malnutrition/chronic inflammation syndrome, and chronic acidosis. Among these predisposing factors; secondary hyperparathyroidism is the most important regarding pathogenesis of tendon rupture. ${ }^{4,14}$ Our patient had more than one predisposing factor of these. He was on long-term hemodialysis since 12years, also he had developed secondary hyperparathyroidism, and his serum b-2microglobulin was $460 \mathrm{mg} / \mathrm{L}$ (normally $<2 \mathrm{mg} / \mathrm{L}$ ).

Diagnosis of rupture tendon in hemodialysis patient starts by clinical examination. Always there is tenderness at the injury site with limited mobility of the affected limb, and a tendon gap can 
be detected. Thompson calf squeeze test is positive for cases with subcutaneous Achilles tendon rupture. Ultrasonography reveals the injury with good sensitivity $(96-100 \%)$ and specificity $(83-100 \%){ }^{15}$ MRI may be helpful when the diagnosis remains unclear, especially in quadriceps tendon rupture. ${ }^{16} \mathrm{It}$ is important to highlight that up to $50 \%$ of quadriceps tendon rupture may be misdiagnosed. Always consider the possibility of a quadriceps tendon rupture in any patient who presents with acute knee pain, an inability to extend the leg, a palpable soft-tissue depression proximal to the superior pole of the patella, and as mentioned before MRI of both thighs may be helpful when the diagnosis remains unclear. ${ }^{16}$ It is also important to highlight that simultaneous, spontaneous, bilateral ruptures may occur, especially of the quadriceps tendon. ${ }^{3}$

Tendon rupture may occur at one of two sites, rupture may occur in the tendon itself due to degenerative changes, or at the tendon insertion site i.e. enthesitis; as secondary hyperparathyroidism increases the osteoclastic cortical bone resorption at the tendon insertion site. ${ }^{17,18}$ Early surgical repair and treatment of ruptured tendons with postoperative physiotherapy and controlling of predisposing factors result in better outcomes than delayed treatment. ${ }^{16}$ Tendon rupture may be a preventable problem by controlling the previous mentioned risk factors as possible.

\section{Conclusion}

Tendon ruptures are uncommon injuries in hemodialysis patients that require early surgical intervention, physiotherapy, with management of predisposing factors in order to maximize functional outcomes for the patient. The most important predisposing factor to be controlled is secondary hyperparathyroidism.

\section{Acknowledgements}

None.

\section{Conflict of interest}

The author declares no conflict of interest.

\section{References}

1. Basic-Jukic N, Juric I, Racki S, et al. Spontaneous tendon ruptures in patients with end-stage renal disease. Kidney Blood Press Res. 2009;32(1):32-36.

2. Park JH, Kim SB, Shin HS, et al. Spontaneous and serial rupture of both Achilles tendons associated with secondary hyperparathyroidism in a patient receiving long-term hemodialysis. Int Urol Nephrol. 2013;45(2):587-590.

3. Jones N, Kjellstrand CM. Spontaneous tendon ruptures in patients on chronic dialysis. Am J Kidney Dis. 1996;28(6):861-866.
4. Palmer S, Birks C, Dunbar J, et al. Simultaneous multiple tendon ruptures complicating a seizure in a haemodialysis patient. Nephrology (Carlton). 2004;9(5):262-264.

5. Gao MF, Yang HL, Shi WD. Simultaneous bilateral quadriceps tendon rupture in a patient with hyperparathyroidism undergoing long-term haemodialysis: a case report and literature review. J Int Med Res. 2013;41(4):1378-1383.

6. Loehr J, Welsh RP. Spontaneous rupture of the quadriceps tendon and patellar ligament during treatment for chronic renal failure. Can Med Assoc J. 1983;129(3):254-256.

7. Wener JA, Schein AJ. Simultaneous bilateral rupture of the patellar tendon and quadriceps expansions in systemic lupus erythematosus. A case report. J Bone Joint Surg Am. 1974;56(4):823-824.

8. Levy M, Seelenfreund M, Maor P, et al. Bilateral spontaneous and simultaneous rupture of the quadriceps tendons in gout. $J$ Bone Joint Surg Br. 1971;53(3):510-513.

9. Razzano CD, Wilde AH, Phalen GS. Bilateral rupture of the infrapatellar tendon in rheumatoid arthritis. Clin Orthop Relat Res. 1973;(91):158161.

10. Bhole R, Johnson JC. Bilateral simultaneous spontaneous rupture of quadriceps tendons in a diabetic patient. South Med J. 1985;78(4):486.

11. Kelly BM, Rao N, Louis SS, et al. Bilateral, simultaneous, spontaneous rupture of quadriceps tendons without trauma in an obese patient:a case report. Arch Phys Med Rehabil. 2001;82(3):415-418.

12. Ho LC, Chiang CK, Huang JW, et al. Rupture of pectoralis major muscle in an elderly patient receiving long-term hemodialysis: case report and literature review. Clin Nephrol. 2009;71(4):451-453.

13. Steiner CA, Palmer LH. Simultaneous bilateral rupture of the quadriceps tendon. Am J Surg. 1949;78(5):752-755.

14. Tsourvakas S, Gouvalas K, Gimtsas C, et al. Bilateral and simultaneous rupture of the triceps tendons in chronic renal failure and secondary hyperparathyroidism. Arch Orthop Trauma Surg. 2004;24(4):278-280.

15. Hartgerink P, Fessell DP, Jacobson JA, et al. Full-versus partial-thickness Achilles tendon tears: sonographic accuracy and characterization in 26 cases with surgical correlation. Radiology. 2001;220(2):406-412.

16. Trobisch PD, Bauman M, Weise $\mathrm{K}$, et al. Histologic analysis of ruptured quadriceps tendons. Knee Surg Sports Traumatol Arthrosc. 2010;18(1):85-88

17. Muratli HH, Celebi L, Hapa O, et al. Simultaneous rupture of the quadriceps tendon and contralateral patellar tendon in a patient with chronic renal failure. J Orthop Sci. 2005;10(2):227-232.

18. Shiota E, Tsuchiya K, Yamaoka K, et al. Spontaneous major tendon ruptures in patients receiving long-term hemodialysis. Clin Orthop Relat Res. 2002;(394):236-242. 